\title{
Precise determination of the strong coupling from energy-energy correlation
}

\section{Zoltán Tulipánt* ${ }^{*}$}

Institute of Physics, University of Debrecen

E-mail: tulipant.zoltan@science.unideb.hu

\section{Adam Kardos $\ddagger$}

Institute of Physics, University of Debrecen

E-mail: kardos.adamescience.unideb.hu

\section{Stefan Kluth}

Max-Planck Institut für Physik

E-mail: stefan.kluth@mpp.mpg • de

\section{Gábor Somogyi}

MTA-DE Particle Physics Research Group, University of Debrecen

E-mail: gabor.somogyi@cern.ch

\section{Andrii Verbytskyi}

Max-Planck-Institut für Physik

E-mail: andrii.verbytskyi@mpp.mpg.de

In this contribution we present a determination of the strong coupling from a fit of QCD predictions for energy-energy correlation measured in electron-positron collisions at various centerof-mass energies. Experimental data are compared to precise theoretical calculations incorporating NNLO QCD predictions combined with NNLL resummation in the back-to-back region. Hadronization corrections are assessed using modern shower Monte Carlo programs matched to NLO fixed-order computations. The final result obtained from a global fit is $\alpha_{\mathrm{S}}\left(M_{Z}\right)=$ $0.11750 \pm 0.0287$ (comb.).

Loops and Legs in Quantum Field Theory (LL2018)

29 April 2018 - 04 May 2018

St. Goar, Germany

\section{${ }^{*}$ Speaker.}

${ }^{\dagger}$ Z.T. was supported by the ÚNKP-17-3 New National Excellence Program of the Ministry of Human Capacities of Hungary.

$\ddagger$ A.K. acknowledges financial support from the Premium Postdoctoral Fellowship program of the Hungarian Academy of Sciences. This work was supported by grant K 125105 of the National Research, Development and Innovation Fund in Hungary. 


\section{Introduction}

Precision measurements of event shape distributions in $e^{+} e^{-}$collisions have provided detailed experimental tests of QCD and remain one of the most precise tools used for extracting the strong coupling $\alpha_{\mathrm{S}}$ from data $[1,2]$. Quantities related to three-jet production are particularly well suited for this task.

The state-of-the-art fixed-order QCD prediction for event shape observables currently includes next-to-next-to-leading order (NNLO) corrections for various quantities [3, 4, 5]. However, fixedorder calculations have a limited range of applicability. For small values of an event shape observable $y$ corresponding to events with two-jet-like configurations the convergence of the fixed-order perturbative series breaks down. This is caused by terms where each power of the strong coupling $\alpha_{\mathrm{S}}^{n}$ is enhanced by a factor of $(\ln y)^{n+1},(\ln y)^{n}$ etc. This problem is addressed with the resummation of such contributions for a fixed number of logarithmic terms. Resummed predictions are known for several event shapes at next-to-next-to-leading log (NNLL) precision while predictions for the C-parameter [6] and thrust [7] are known up to $\mathrm{N}^{3} \mathrm{LL}$ accuracy.

So far the measurements of the strong coupling based on $e^{+} e^{-}$annihilation use either jet rates or event shapes describing global topology (e.g. thrust, C-parameter), although one can also consider observables based on particle correlations like the energy-energy correlation (EEC) for determining $\alpha_{\mathrm{S}}$ from three jet production.

\section{Energy-energy correlation}

EEC is the normalized energy-weighted cross section defined in terms of the angle between two particles $i$ and $j$ in an event [8]:

$$
\frac{1}{\sigma_{t}} \frac{d \Sigma}{d \cos \chi}=\frac{1}{\sigma_{t}} \int \sum_{i, j} \frac{E_{i} E_{j}}{Q^{2}} d \sigma_{e^{+} e^{-} \rightarrow i j+X} \delta\left(\cos \chi-\cos \theta_{i j}\right),
$$

where $E_{i}$ and $E_{j}$ are the particle energies, $Q$ is the center-of-mass energy, $\theta_{i j}=\chi$ is the angle between the three-momenta of the two particles and $\sigma_{t}$ is the total hadronic cross section. The back-to-back region $\theta_{i j} \rightarrow 180^{\circ}$ corresponds to $\chi \rightarrow \pi$.

The EEC was extensively measured at LEP, PEP, PETRA, SLC and TRISTAN at various center-of-mass energies.

\section{Fixed-order and resummed calculations}

We performed fixed-order perturbative calculations up to NNLO accuracy using the CoLoRFulNNLO scheme $[5,9,10]$ as implemented in the MCCSM code [11]. At the default renormalization scale of $\mu=Q$ the fixed-order prediction reads

$$
\left[\frac{1}{\sigma_{t}} \frac{d \Sigma}{d \cos \chi}\right]_{(\text {f.o. })}=\frac{\alpha_{\mathrm{S}}}{2 \pi} \frac{d \bar{A}}{d \cos \chi}+\left(\frac{\alpha_{\mathrm{S}}}{2 \pi}\right)^{2} \frac{d \bar{B}}{d \cos \chi}+\left(\frac{\alpha_{\mathrm{S}}}{2 \pi}\right)^{3} \frac{d \bar{C}}{d \cos \chi}+\mathscr{O}\left(\alpha_{\mathrm{S}}^{4}\right)
$$

where $\bar{A}, \bar{B}$ and $\bar{C}$ are the perturbative coefficients at LO, NLO and NNLO, normalized to the total cross section for $e^{+} e^{-} \rightarrow$ hadrons, $\sigma_{t}$. 
The fixed-order perturbative predictions diverge for both small and large values of $\chi$, due to the presence of large logarithmic contributions of infrared origin. In the back-to-back region $\chi \rightarrow \pi$, these contributions take the form $\alpha_{S}^{n} \log ^{2 n-1} y$, where

$$
y=\cos ^{2} \frac{\chi}{2} \text {. }
$$

As $y$ increases, the logarithms become large and invalidate the use of the fixed-order expansion. We can obtain a description of EEC in this limit by resumming the logarithmic contributions to all orders. This resummation has been computed at NNLL accuracy in Ref. [12] while in Ref. [13] a factorization theorem for EEC was derived based on soft-collinear effective theory which will allow to perform the resummation at $\mathrm{N}^{3} \mathrm{LL}$ accuracy once the corresponding NNLO jet function is computed. Since the complete jet function is currently unavailable, we use the NNLL results and formalism of Ref. [12].

For combining the two perturbative calculations we performed log-R matching. However, the fixed-order expansion of the EEC diverges for both small and large angles making the determination of a simple cumulant unreliable. Hence, we use a linear combination of moments to suppress the singularity at $\chi=\pi$

$$
\frac{1}{\sigma_{t}} \tilde{\Sigma}(\chi) \equiv \frac{1}{\sigma_{t}} \int_{0}^{\chi} d \chi^{\prime}\left(1-\cos \chi^{\prime}\right) \frac{d \Sigma}{d \chi^{\prime}} .
$$

Log-R matching is performed for the quantity $\tilde{\Sigma}$ and we can recover $\Sigma$ by

$$
\frac{1}{\sigma_{t}} \frac{d \Sigma}{d \chi}=\frac{1}{1-\cos \chi} \frac{1}{\sigma_{t}} \frac{d \tilde{\Sigma}}{d \chi} .
$$

For the details of the matching procedure see Ref. [14].

\section{Finite b-quark mass corrections and hadronization effects}

The fixed-order NNLO prediction and the resummation were computed in massless QCD. However, at low energies the assumption of vanishing quark masses is not entirely justified. Hence, we included b-quark mass effects directly at the level of matched distributions

$$
\frac{1}{\sigma_{t}} \frac{d \Sigma(\chi, Q)}{d \cos \chi}=\left(1-r_{b}(Q)\right)\left[\frac{1}{\sigma_{t}} \frac{d \Sigma(\chi, Q)}{d \cos \chi}\right]_{\text {massless }}^{N N L O+N N L L}+r_{b}(Q)\left[\frac{1}{\sigma_{t}} \frac{d \Sigma(\chi, Q)}{d \cos \chi}\right]_{\text {massive }}^{N N L O *}
$$

where $r_{b}(Q)$ denotes the fraction of b-quark events which we defined as

$$
r_{b}(Q) \equiv \frac{\sigma_{\text {massive }}\left(e^{+} e^{-} \rightarrow b \bar{b}\right)}{\sigma_{\text {massive }}\left(e^{+} e^{-} \rightarrow \text { hadrons }\right)} .
$$

The complete NNLO correction to the massive distribution is unknown, thus we supplement the massive NLO prediction of Zbb4 [15] with the NNLO coefficient of the massless calculation (denoted as $N N L O *$ above). Distributions were generated for each $Q$ separately assuming a pole b-quark mass of $m_{b}=4.75 \mathrm{GeV}$.

Non-perturbative corrections were taken into account using particle-level Monte Carlo event generators to compute point-by-point multiplicative correction factors. This procedure simultaneously allows for the extraction of the missing statistical correlations of data points. Hadronization 
corrections were determined as ratios of hadron to parton level distributions in the Monte Carlo simulations. The simulated samples were reweighted to data at hadron level on an event-by-event basis to assure a better description of data. To tame the statistical fluctuations present in the obtained corrections, these were parametrized with analytic functions. This parametrization is valid only inside the fit range. In our analysis four setups were used:

- $S^{L}$ : default setup, Sherpa2 .2 . 4 [19], using Lund string model

- $S^{C}$ : for hadronization systematics, Sherpa2 .2.4, using cluster hadronization

- $H^{M}$ : for MC cross check, Herwig7 .1.1 [20], using cluster hadronization

- $D M W$ : for cross check with the analytic hadronization model of Ref. [16]

\section{Fit procedure and results}

To extract the strong coupling the predictions were confronted with the data obtained in SLD [21], L3 [22], DELPHI [23], OPAL [24, 25], TOPAZ [26], TASSO [27], JADE [28], MAC [29], MARKII [30], CELLO [31] and PLUTO [32] experiments. The criteria to include the data were high precision of differential distributions obtained with charged and neutral final state particles in the full kinematic range, presence of corrections for detector effects, correction for initial state photon radiation and sufficient amount of supplementary information. Hence, datasets without supplementary information [33], with large uncertainties [34], superseded datasets [35, 36] and measurements unfolded only to charged particles in the final state [37] are not included in the analysis.

The strong coupling extraction is based on the comparison of data to the perturbative QCD prediction combined with non-perturbative corrections. To find the optimal value of $\alpha_{S}$, the MINUIT2 $[17,18]$ program was used to minimize

$$
\chi^{2}\left(\alpha_{\mathrm{S}}\right)=\sum_{\text {datasets }} \chi^{2}\left(\alpha_{\mathrm{S}}\right)_{\text {dataset }},
$$

where $\chi^{2}\left(\alpha_{\mathrm{S}}\right)$ was calculated for each dataset as

$$
\chi^{2}\left(\alpha_{\mathrm{S}}\right)=\left(\vec{D}-\vec{P}\left(\alpha_{\mathrm{S}}\right)\right) V^{-1}\left(\vec{D}-\vec{P}\left(\alpha_{\mathrm{S}}\right)\right)^{T},
$$

with $\vec{D}$ standing for the vector of data points, $\vec{P}\left(\alpha_{\mathrm{S}}\right)$ for the vector of calculated points and $V$ for the covariance matrix for $\vec{D}$.

The fit ranges were chosen to avoid regions where either the resummed prediction or the hadronization corrections are unreliable. The selected fit range was $\left[60^{\circ}, 160^{\circ}\right]$ and we have observed that the final results are insensitive to a $\pm 5^{\circ}$ change in the fit range. Representative fit results are shown in Fig. 1.

To estimate the bias caused by neglecting higher-order terms in the perturbative predictions, variation of renormalization scale (ren.) in the range $x_{R}=\mu_{R} / Q \in[1 / 2,2]$ and variation of resummation scale (res.) in the range $x_{L} \in[1 / 2,2]$ were performed as show in Fig. 2. The fits were repeated by varying the scales $x_{R}$ and $x_{L}$ independently. The bias of the hadronization model selection ( $h a d r$.) was studied using the $S^{L}$ and $S^{C}$ setups of non-perturbative corrections. The uncertainty 

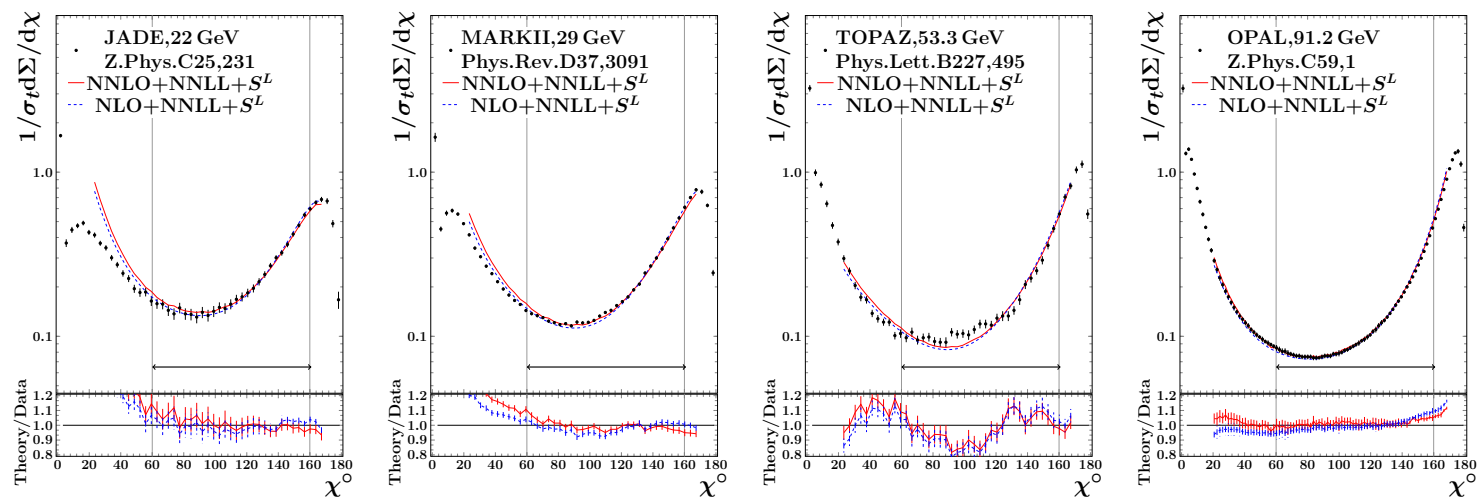

Figure 1: Representative fits of the theoretical predictions to measured data. The fit range is indicated by thick lines.
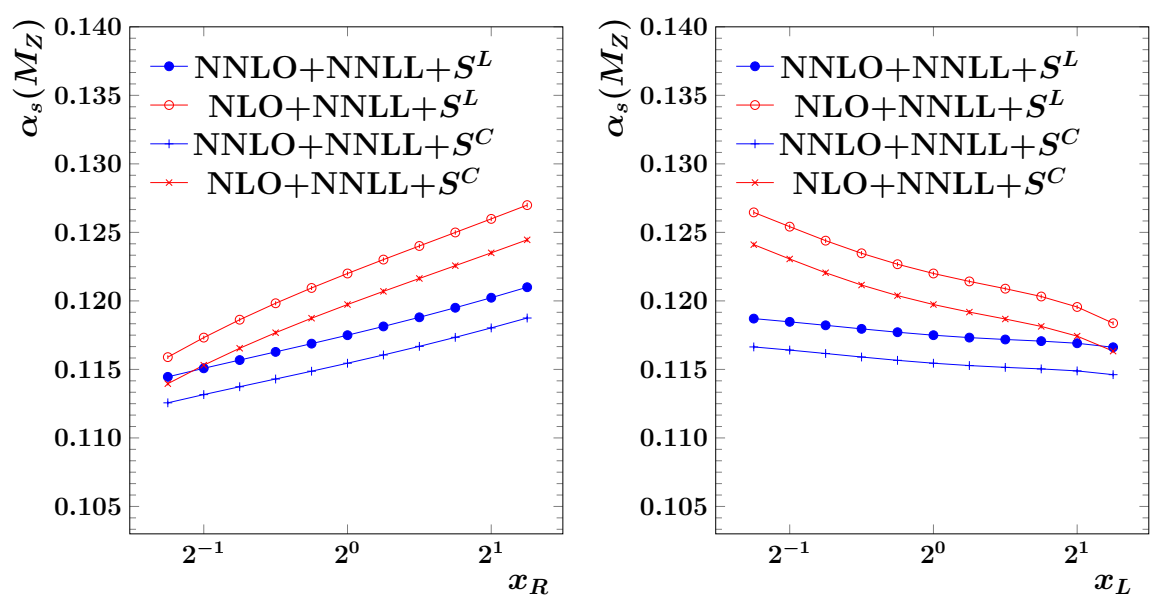

Figure 2: Dependence of fit results on the renormalization scale $x_{R}$ and resummation scale $x_{L}$.

on the fit result (exp.) was obtained with the $\chi^{2}+1$ criterion as implemented in MINUIT2. As a cross check the datasets were grouped according to their energies and fitted separately for each energy. See Fig. 3. These results show no visible trend for the fitted value of $\alpha_{\mathrm{S}}$ with energy in the $S^{L}$ and $S^{C}$ setups.

For the central value of the final result we quote the results from the fits with the $S^{L}$ hadronization model in the range $\left[60^{\circ}, 160^{\circ}\right]$ with uncertainties obtained as described above. At NNLO+NNLL accuracy the global fit yielded the value of

$$
\alpha_{\mathrm{S}}\left(M_{Z}\right)=0.11750 \pm 0.00018(\text { exp. }) \pm 0.00102 \text { (hadr.) } \pm 0.00257 \text { (ren.) } \pm 0.00078 \text { (res.). }
$$

In order to assess the impact of NNLO corrections, we also quote the result of a global fit performed at NLO+NNLL accuracy

$$
\alpha_{\mathrm{S}}\left(M_{Z}\right)=0.12200 \pm 0.00023(\text { exp. }) \pm 0.00113 \text { (hadr.) } \pm 0.00433 \text { (ren.) } \pm 0.00293 \text { (res.). }
$$

It is apparent that the inclusion of the NNLO corrections has a moderate but non-negligible effect on the extracted value of $\alpha_{\mathrm{S}}$. It has been explicitly checked that there are no correlations between the 

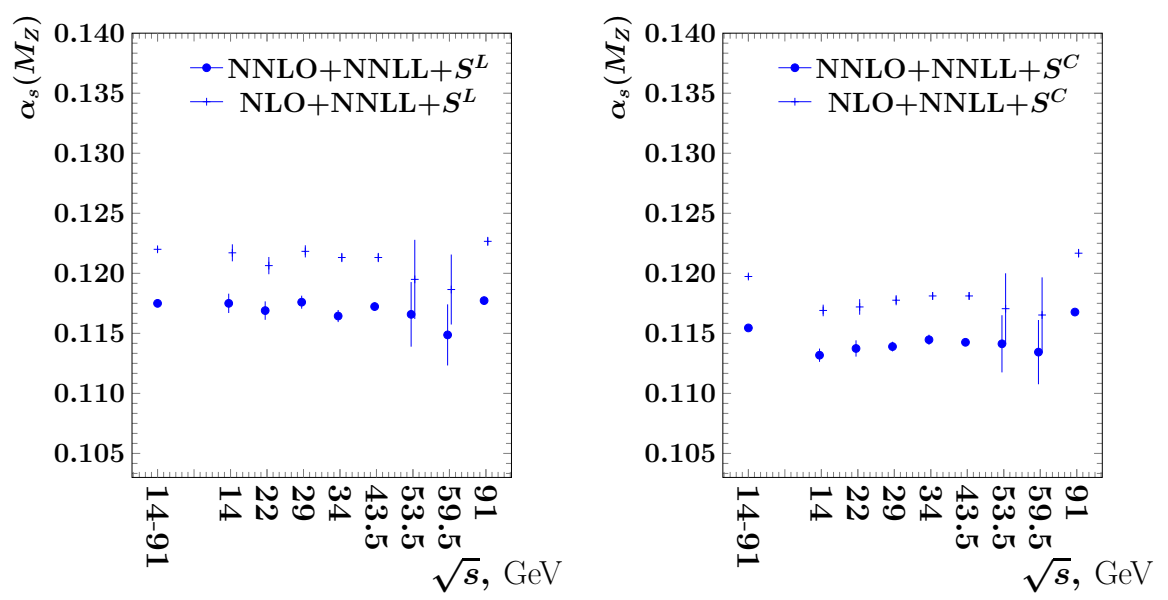

Figure 3: Dependence of fit results on the used datasets.

estimated biases, therefore, the value with combined estimations of uncertainty at NNLO+NNLL accuracy is

$$
\alpha_{\mathrm{S}}\left(M_{Z}\right)=0.11750 \pm 0.00287(\mathrm{comb} .)
$$

while in comparison, for NLO+NNLL accuracy we have

$$
\alpha_{\mathrm{S}}\left(M_{Z}\right)=0.12200 \pm 0.00535(\text { comb. }) \text {. }
$$

The estimated uncertainties are dominated by the uncertainty of the theoretical prediction, mostly by the fixed-order calculation (ren.).

A further cross check was performed with the $D M W$ analytic hadronization setup. In this procedure, non-perturbative effects were modelled by applying a multiplicative correction to the Sudakov form factor which contains two additional parameters to be fitted. For details see Ref. [16]. We have found that the values obtained for $\alpha_{\mathrm{S}}\left(M_{Z}\right)$ in the range $\left[117^{\circ}, 165^{\circ}\right]$ are close to the values obtained using the Monte Carlo hadronization setups but depend strongly on the choice of fit range. Hence, we conclude that the analytic model cannot fully describe hadronization away from the back-to-back region.

\section{Conclusions}

A new measurement of $\alpha_{\mathrm{S}}\left(M_{Z}\right)$ was presented using a global fit of EEC in $e^{+} e^{-}$collisions to NNLO+NNLL QCD predictions. Our analysis yielded the final value of

$$
\alpha_{\mathrm{S}}\left(M_{Z}\right)=0.11750 \pm 0.00287(\mathrm{comb} .)
$$

The inclusion of NNLO corrections resulted in a better modelling of the shape of the distribution and a non-negligible shift of the extracted $\alpha_{\mathrm{S}}\left(M_{Z}\right)$ towards lower values. Furthermore, theoretical uncertainties were considerably reduced. Our result is in agreement with the world average as of 2017 [38] and the combined uncertainty is competitive with the uncertainties of the other analyses based on $e^{+} e^{-}$annihilation. 
Considering the latest advances towards further improving the perturbative description with analytic NLO corrections $[39,40]$ and $N^{3}$ LL resummation it will be interesting to revisit this topic when the resummed prediction becomes fully available.

\section{References}

[1] S. Kluth, Tests of quantum chromo dynamics at $e^{+} e^{-}$colliders, Rept. Prog. Phys. 69, 1771 (2006)

[2] G. Dissertori, The determination of the strong coupling constant, Adv. Ser. Direct. High Energy Phys. 26, 113 (2016)

[3] A. Gehrmann-De Ridder et al., NNLO corrections to event shapes in $e^{+} e^{-}$annihilation, JHEP 12, 094 (2007)

[4] S. Weinzierl, Event shapes and jet rates in electron-positron annihilation at NNLO, JHEP 06, 041 (2009)

[5] V. Del Duca et al., Jet production in the CoLoRFulNNLO method: event shapes in electron-positron collisions, Phys. Rev. D94, 074019 (2016)

[6] A. Hoang et al., C-parameter distribution at $\mathrm{N}^{3} \mathrm{LL}$ ' including power corrections, Phys. Rev. D91, 094017 (2015)

[7] R. Abbate et al., Thrust at $\mathrm{N}^{3} \mathrm{LL}$ with power corrections and a precision globat ir for $\alpha_{\mathrm{S}}\left(M_{Z}\right)$, Phys. Rev. D83, 074021 (2011)

[8] C. Basham et al., The energy-energy correlation in electron-positron annihilation: testing QCD, Phys. Rev. Lett. 41, 1585 (1978)

[9] G. Somogyi, Z. Trócsányi and V. Del Duca, A subtraction scheme for computing QCD jet cross sections at NNLO: Regularization of doubly-real emissions, JHEP 01, 070 (2007)

[10] G. Somogyi and Z. Trócsányi, A subtraction scheme for computing QCD jet cross sections at NNLO: Regularization of the real-virtual emission, JHEP 01, 052 (2007)

[11] A. Kardos, G. Somogyi and Z. Trócsányi, Jet cross sections with CoLoRFulNNLO, PoS LL2016, 021 (2016)

[12] D. de Florian and M. Grazzini, The back-to-back region in $e^{+} e^{-}$energy-energy correlation, Nucl Phys. B704, 387 (2005)

[13] I. Moult and Hua Xing Zhu, Simplicity from Recoil: The Three-Loop Soft Function and Factorization for the Energy-Energy Correlation, (2018), arXiv:1801.02627

[14] Z. Tulipánt, A. Kardos and G. Somogyi, Energy-energy correlation in electron-positron annihilation at NNLL+NNLO accuracy, Eur. Phys. J C77, 749 (2017)

[15] P. Nason and C. Oleari, Next-to-leading order corrections to momentum correlations in $Z^{0} \rightarrow b \bar{b}$, Phys. Lett. B407, 57 (1997)

[16] Y. L. Dokshitzer, G. Machesini and B. R. Webber, Nonperturbative effects in the energy energy correlation, JHEP 12, 007 (2003)

[17] F. James and M. Roos, Minuit: A system for function minimization and analysis of the parameter errors and correlations, Comput. Phys. Commun. 10, 343 (1975)

[18] F. James and M. Winkler, MINUIT User's Guide, (2004) 
[19] T. Gleisberg et al., Event generation with SHERPA 1.1, JHEP 02, 007 (2009)

[20] J. Bellm et al., Herwig 7.0/Herwig++ 3.0 release note, Eur. Phys. J. C76, 196 (2016)

[21] SLD Coll., K. Abe et al., Measurement of $\alpha_{\mathrm{S}}\left(M_{Z}\right)$ from hadronic event observables at the $Z^{0}$ resonance, Phys. Rev. D51, 962 (1995)

[22] L3 Coll., O. Adrian et al., Determination of $\alpha_{\mathrm{S}}$ from hadronic event shapes measured on the $Z^{0}$ resonance, Phys. Lett. B284, 471 (1992)

[23] DELPHI Coll., P. Abreu et al., Determination of $\alpha_{\mathrm{S}}$ in second order QCD from hadronic $Z$ decays, Z. Phys. C54, 55 (1992)

[24] OPAL Collaboration, P. D. Acton et al., A determination of $\alpha_{\mathrm{S}}\left(M_{Z}\right)$ at LEP using resummed QCD calculations, Z. Phys. C59, 1 (1993)

[25] OPAL Coll., P. D. Acton et al., An Improved measurement of $\alpha_{\mathrm{S}}\left(M_{Z}\right)$ using energy correlations with the OPAL detector at LEP, Phys. Lett. B276, 547 (1992)

[26] TOPAZ Coll., I. Adachi et al., Measurements of $\alpha_{\mathrm{S}}$ in $e^{+} e^{-}$annihilation at $\sqrt{s}=53.3 \mathrm{GeV}$ and 59.5GeV, Phys. Lett. B227, 495 (1989)

[27] TASSO Coll., W. Braunschweig et al., A study of energy-energy correlations between $12 \mathrm{GeV}$ and 46.8GeV CM energies, Z. Phys. C36, 349 (1987)

[28] JADE Coll., W. Bartel et al., Measurements of energy correlations in $e^{+} e^{-} \rightarrow$ hadrons, Z. Phys. C25, 231 (1984)

[29] MAC Coll., E. Frenandez et al., A measurement of energy-energy correlations in $e^{+} e^{-} \rightarrow$ Hadrons at $\sqrt{s}=29 \mathrm{GeV}$, Phys. Rev. D31, 2724 (1985)

[30] MARKII Coll., D. R. Wood et al., Determination of $\alpha_{\mathrm{S}}$ from energy-energy correlations in $e^{+} e^{-}$ annihilation as 29GeV, Phys. Rev. D37, 3091 (1988)

[31] CELLO Coll., H. J. Behrend et al., Analysis of the energy weighted angular correlations in hadronic $e^{+} e^{-}$annihilations at $22 \mathrm{GeV}$ and $34 \mathrm{GeV}$, Z. Phys. C14, 95 (1982)

[32] PLUTO Coll., C. Berger et al., A study of energy-energy correlations in $e^{+} e^{-}$annihilations at $\sqrt{s}=34.6 \mathrm{GeV}, \mathrm{Z}$. Phys. C28, 365 (1985)

[33] PLUTO Coll., C. Berger et al., Energy-energy correlations in $e^{+} e^{-}$annihilation into hadrons, Phys. Lett 99B, 292 (1981)

[34] L3 Coll., B. Adeva et al., Determination of $\alpha_{\mathrm{S}}$ from energy-energy correlations measured on the Z0 resonance, Phys. Lett. B257, 469 (1991)

[35] OPAL Coll., M. Z. Akrawy et al., A measurement of energy correlations and a determination of $\alpha_{\mathrm{S}}\left(M_{Z^{0}}\right)$

[36] SLD Coll., K. Abe et al., Measurement of $\alpha_{\mathrm{S}}$ from energy-energy correlation at the $Z^{0}$ resonance, Phyr. Rev. D50, 5580 (1994)

[37] DELPHI Coll., P. Abreu et al., Consistent measurements of $\alpha_{\mathrm{S}}$ from precise oriented event shape distributions, Eur. Phys. J. C14, 557 (2000)

[38] S. Bethke, $\alpha_{\mathrm{S}}$ 2016, Nucl. Part. Phys. Proc. 282-284, 149 (2017)

[39] L. J. Dixon et al., Analytical Computation of Energy-Energy Correlation Next-to-Leading Order in QCD, Phys. Rev. Lett. 120, 102001 (2018)

[40] O. Gituliar and S. Moch, Fuchsia and Master Integrals of Energy-Energy Correlation at NLO in QCD, Acta Phys. Polon. B48, 2355 (2017) 